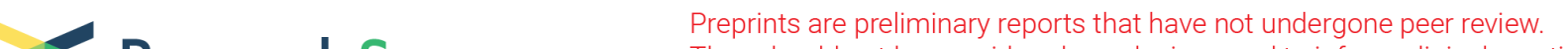 $\begin{array}{ll}\text { Research Square } & \begin{array}{l}\text { They should not be considered conclusive, used to inform clinical practice, } \\ \text { or referenced by the media as validated information. }\end{array}\end{array}$
}

\section{Time to Focus on the Scalp Incision Design for Chronic Subdural Hematoma: Technical Note and Experience}

\section{Juexian Xiao}

The Second Affiliated Hospital of Nanchang University

Yu Liu (Dly516999@163.com )

Nanchang University Second Affiliated Hospital https://orcid.org/0000-0002-6405-0141

Shikai Gui

The Second Affiliated Hospital of Nanchang University

\section{Feng Wei}

The Second Affiliated Hospital of Nanchang University

\section{Tong Zhou}

The Second Affiliated Hospital of Nanchang University

\section{Shulong LV}

The Second Affiliated Hospital of Nanchang University

\section{Peng Chen}

The Second Affiliated Hospital of Nanchang University

\section{Xingen Zhu}

The Second Affiliated Hospital of Nanchang UniversityThe Second Affiliated Hospital of Nanchang University

\section{Zujue cheng}

The Second Affiliated Hospital of Nanchang University https://orcid.org/0000-0003-2490-7507

\section{Research Article}

Keywords: Chronic subdural hematoma, Scalp incision, Cosmetic dissatisfaction

Posted Date: September 27th, 2021

DOI: https://doi.org/10.21203/rs.3.rs-891241/v1

License: (c) (i) This work is licensed under a Creative Commons Attribution 4.0 International License. Read Full License 


\section{Abstract}

Objective: The scalp incision design in surgery for chronic subdural hematoma (CSDH) is important but is given little attention or is neglected by most neurosurgeons currently. Faulty scalp incision design will affect the ease of surgery, wound healing, and patients' satisfaction. This study introduces the technique and experience used in our center regarding scalp incision with an adjustable question mark-shaped for CSDH procedures.

Methods: A retrospective analysis was conducted of the medical records of patients with CSDH, who underwent surgical treatment that incorporated this incision design from September 2017 through December 2020. The features of concern were intraoperative scalp incision extension and postoperative wound healing. Those patients were followed for occurrences of scalp dysfunction, and their satisfaction with this design.

Results: Thirty-seven patients were analyzed. For 2 patients, operative procedures were changed and scalp incisions were extended. All patients had good incision healing without complications. The outpatient or telephone follow-up 6 months after surgery revealed that 36 patients were satisfied with the scalp incision design, one patient experienced widening of incision scar. Six patients ( 5 women and 1 man) reported cosmetic dissatisfaction, and 7 patients (six women and one man) had mild scalp dysfunction, in daily life.

Conclusion: The adjustable question mark-shaped incision used for CSDHs is a fluent curve, with a large angle, and allows a flexible extension. It ensures ease of the operative procedure, good wound healing and meeting patients' aesthetic needs. The protocol is simple, practical, and worthy of reference by neurosurgeons, especially in primary hospitals.

\section{Introduction}

The Seventh National Population Census of the People's Republic of China in 2020 showed that China has the largest population in the world, and the population is quickly ageing(http://www.stats.gov.cn/english/PressRelease/202105/t20210510_1817186.html). Therefore, in China, the number of patients with chronic subdural hematoma (CSDH), which is prone to the elderly, is very large, although there are few demographic descriptions about Chinese patients with CSDH [1]. Surgical intervention is required for patients with CSDH whose quality of life had been affected severely and whose symptoms continue to worsen after conservative treatments. Burr hole (single- or double-hole) craniotomy and mini-craniotomy are common procedures for CSDHs [2-4].

Clinical studies on CSDHs mainly focus on improvements in surgical technique, the reduction of complications, and the recurrence rate of hematoma. Very few researchers have focused on the scalp incision design for CSDHs. Faulty or poor scalp incision design will confound the surgery and risk scalp healing complications such as necrosis and dehiscence [5]. This degrades the surgical outcome, prolongs hospitalization, increases healthcare costs, and aggravates the physical and psychosocial distress of the 
patients. An ill-considered scalp incision can also cause aesthetic defects [6], and some patients may experience cosmetic self-consciousness that seriously affects their quality of daily life [7].

Unfortunately, scalp incision design for CSDH can be considered unimportant and neglected by some neurosurgeons. No specialized studies have focused on incision design for CSDHs. To provide a reference for neurosurgeons, this study introduces an adjustable question mark-shaped scalp incision for use during CSDH procedures. This design is based on experience at our center. The article includes a detailed discussion of the principle and method.

\section{Methods}

\section{Patient identification}

The medical data were retrospectively collected of patients with CSDH treated with an adjustable question mark-shaped incision between September 2017 and December 2020. All patients had their first surgery, and their scalps were in good health. All patients were postoperatively followed for 6 months by outpatient or telephone enquiry. Those who did not follow up were excluded at the sixth month. The status of the scalp incision healing and patients' satisfaction with the design of the scalp incision were included in the follow-up study.

\section{Design protocol of the adjustable question mark-shaped scalp incision}

On the affected side, a question mark-shaped line is usually drawn on the frontal-parietal-temporal scalp (Fig. 1). This line resembles the line used in decompressive hemicraniectomy, but is smaller. The anterior half of the question-mark is roughly parallel to the superior temporal line, and its front end intersects the scalp projection of the coronal suture and ends within the hairline of the forehead. The posterior end, that is, the arc of the question mark, is located at the parietal tubercle or about 0.5- to 1-centimeter anteroinferior to the parietal tubercle. Taking the arc as the reference, according to the location and size of the hematoma, a surgeon can adjust the question-mark line forward, backward, or up or down as needed, to make the main part pass through the projection area of the hematoma cavity.

For single burr-hole craniotomy, a 2- to 3-centimeter incision is made along the arc line (the posterior end of the question-mark; Fig. 2). In double burr-hole craniotomy, the other incision is placed at the intersection of the question-mark and the projection of the coronal suture, or slightly anterior to the coronal suture (Fig. 3). In 3-dimensional space, the positions of the 2 incisions are formed anterior-toposterior and high-to-low to facilitate the double hole irrigation during the operation, to irrigate the hematoma completely. When a surgeon performs a mini-craniotomy, the incision is also located on the arc part of the question-mark, and the radian of the arc can be reduced moderately to make the exposed skull about 2.5- to 3-centimeters in diameter (Fig. 4). Sometimes, the arc-shaped incision line can be appropriately moved forward (Fig. 5). If the hematoma is difficult to drain, the scalp incision can be extended along the adjustable question mark-shaped line and a large craniotomy can be performed. 


\section{Statistical analysis}

Baseline characteristics of this study were summarized using descriptive statistics, by mean \pm standard deviation, or $\mathrm{n}(\%)$. The independent $t$-test and Kruskal-Wallis $H$ test were performed to compare continuous variables. The chi-squared test and Fisher's exact test were performed to compare categorical variables. Univariate logistic regression analysis was used to estimate the odds ratios (ORs) and 95\% confidence intervals (Cls). A P value less than 0.05 was considered statistically significant. All analyses were performed using EmpowerStats (http://www.empowerstats.com) and the statistical package R.

\section{Results}

The study population consisted of 27 men (72.97\%) and 10 women (27.03\%), aged $64.86 \pm 6.09$ years (Table 1). The CSDH was left hemispheric in 18 patients (48.65\%), right hemispheric in 13 patients (35.14\%) and bilateral in 6 patients (16.22\%). Nineteen (51.35\%) and 18 patients (48.65\%) underwent burr-hole and mini-craniotomy, respectively. During mini-craniotomy, there was difficulty with drainage of the hematoma in 2 of the patients (one sediment hematoma and one multi-fibrous compartmented hematoma), and scalp incisions were extended along the adjustable question mark-shaped line to remove the hematoma with a large craniotomy. 
Table 1

Baseline characteristics of the study participants and followup outcomes *

\begin{tabular}{|lll|}
\hline Age, $\mathbf{y}$ & & $64.86 \pm 6.09$ \\
\hline Gender & Male & $27(72.97)$ \\
\hline Side of hematoma & Female & $10(27.03)$ \\
\hline & Left & $18(48.65)$ \\
\hline Craniotomy & Bilateral & $13(35.14)$ \\
\hline & One-burr hole & $13(35.14)$ \\
\hline Scalp incision extending & No & $6(16.22)$ \\
\hline Status of incision & Mini & $18(48.65)$ \\
\hline Satisfaction with incision & No & $35(94.59)$ \\
\hline Kood healing & $37(100.00)$ \\
\hline Cosmetic dissatisfaction & No & $1(2.70)$ \\
\hline Functional handicaps & No & $36(97.30)$ \\
\hline & Yes & $6(16.22)$ \\
\hline & $30(81.08)$ \\
\hline & $7(18.92)$ \\
\hline
\end{tabular}

All patients had good wound healing without any complications, including necrosis or dehiscence. Outpatient or telephone follow-up surveys were performed at 6 months postoperatively. Thirty-six patients reported that they were satisfied with the adjustable question mark-shaped line design, except for one woman with widening of the incision scar. Six patients ( 5 for burr-hole craniotomy, and one for minicraniotomy; 5 women and 1 man) were mildly self-consciousness of a slightly depressed scalp and for other cosmetic reasons.

The mean age of patients who reported cosmetic dissatisfaction was significantly younger than those who were not troubled in this way, and the difference was statistically significant (59.50 $\pm 4.51 \mathrm{cf} .65 .90 \pm$ 5.85 ; OR $1.23,95 \% \mathrm{Cl} 0.31-2.14$, respectively, $\mathrm{P}=0.016$; Table 2 ). Significantly more women than men reported self-consciousness for cosmetic reasons ( 5 women and one man; OR 1.82, 95\% Cl 0.85-2.78, P 
<0.001). Seven patients ( 6 women and $1 \mathrm{man}$ ) had mild scalp dysfunction in daily life, manifested as mild discomfort of the scalp incision when combing or washing hair, or itchy scalp (Table 3). Significantly more women than men reported such functional handicaps $(P<0.001)$.

Table 2

Distribution of cosmetic dissatisfaction by age, gender, and surgical procedure *

\begin{tabular}{|llllll|}
\hline & & No & Yes & OR $(95 \%$ Cl $)$ & $P$ \\
\hline Age, y & & $65.90 \pm 5.85$ & $59.50 \pm 4.51$ & $1.23(0.31,2.14)$ & 0.016 \\
\hline Gender & Male & $26(83.87)$ & $1(16.67)$ & $1.82(0.85,2.78)$ & $<0.001$ \\
\hline & Female & $5(16.13)$ & $5(83.33)$ & & \\
\hline Craniotomy & One-burr hole & $10(32.26)$ & $3(50.00)$ & $0.89(-0.00,1.79)$ & 0.197 \\
\hline & Two-burr hole & $4(12.90)$ & $2(33.33)$ & & \\
\hline & Mini & $17(54.84)$ & $1(16.67)$ & & \\
\hline
\end{tabular}

Table 3

Distribution of functional handicaps by age, gender, and surgery

\begin{tabular}{|llllll|}
\hline & & No & Yes & OR $(95 \% \mathrm{Cl})$ & $P$ \\
\hline Age & & $65.03 \pm 6.37$ & $64.14 \pm 5.08$ & $0.15(-0.67,0.98)$ & 0.733 \\
\hline Gender & Male & $26(86.67)$ & $1(14.29)$ & $2.10(1.15,3.05)$ & $<0.001$ \\
& Female & $4(13.33)$ & $6(85.71)$ & & \\
\hline Craniotomy & One-burr hole & $12(40.00)$ & $1(14.29)$ & $0.65(-0.18,1.49)$ & 0.366 \\
\hline & Two-burr hole & $5(16.67)$ & $1(14.29)$ & & \\
\hline & Mini & $13(43.33)$ & $5(71.43)$ & & \\
\hline
\end{tabular}

\section{Discussion}

A practical scalp incision design is directly related to ease of procedure and surgical effect, and is a prerequisite for the successful surgery of CSDHs. The ideal scalp incision design for CSDH surgery should satisfy the following 4 criteria: The incision should be positioned to facilitate drainage of the hematoma and provide the most flexible option for intraoperative incision extension. Moreover, the scalp incision should heal well, and satisfy the patient's desire for an aesthetic incision [8, 9]. 
Selection of the site of the incision has no standard rules or criteria, either for burr hole craniotomy or mini-craniotomy; selection is mainly based on the location and volume of the CSDHs. Clinically, the volume of a CSDH that requires surgical intervention is usually large, and most of the hematoma accumulates in the frontotemporal and parietal regions. Hematoma can be drained in any position of the projected scalp of the hematoma cavity. However, if the incision is below the superior temporal line, part of the temporal muscle is bound to be dissected, which causes different degrees of postoperative temporal muscle injury and edema, and even atrophy in severe cases. These may cause pain, weakness, or other annoyances during chewing.

If the incision is too far below the superior temporal line, the middle meningeal artery or its thick branches may be encountered during the procedure. The procedure with improperly handled will result in postoperative complications such as acute subdural or epidural hematoma. In serious cases, a second operation is required. With our long-term clinical experience, we found that the scalp projection of the main anterior part of the hematoma cavity is in the coronal suture or slightly anterior to it, and that of the posterior part of the hematoma cavity is approximately at the parietal tubercle, or about $0.5-$ to 1centimeter anteroinferior to the parietal tubercle. These two areas are at the same computed tomography level, roughly. Setting the incision at the above two locations can direct hematoma drainage and effectively avoid potential complications. In addition, for a single burr-hole craniotomy, we recommend that the incision should be located at 0.5- to 1-centimeter anteroinferior to the parietal tubercle. This low location facilitates drainage and thus reduces the recurrence rate of hematoma.

After the site is selected, the incision shape should be strategized. There are no standard guidelines, and shape can depend on the preference of the operator. All factors should be considered, including extensibility, blood supply, and aesthetic appearance. Each of these is discussed below.

\section{Extensibility of the scalp incision shape}

Clinically, most CSDHs can be successfully drained during surgery. However, difficulties may be encountered in some cases such as sediment hematoma, multi-fibrous compartmented hematoma, and calcified hematoma. For these types of hematomas, the scalp incision should be extended, and a large craniotomy should be performed to remove the hematoma.

A procedure is also needed when there is severe active bleeding, but the site of bleeding cannot be explored. Additionally, the possibility that the incision may have to be reused should be considered in the design of its shape; the patient may need reoperation for hematoma recurrence or other craniocerebral diseases later in life. Consequently, if the designed incision shape cannot be flexibly extended, subsequent procedures will not go smoothly or even fail.

\section{Scalp incision shape and blood supply}


After the position is selected, the incision shape is related to the blood supply of the scalp incision, and directly affects the postoperative wound healing. Some authors have reported that the " $T$ " or " $n$ " incision shapes, especially in patients with diabetes or drug abuse, are more prone to ischemia and necrosis, leading to wound healing complications $[10,11]$. In our clinical experience, when the angle formed by the incision lines, or lines tangent to the incision are less than or equal to 90 degrees, then the incision, especially the corner of the incision, is prone to ischemia and necrosis. Therefore, a scalp incision that is smooth and wider than 90 degrees is a powerful guarantee for good wound healing.

\section{Aesthetic appearance of the scalp incision shape}

Patients are concerned about the cosmetic results of surgeries, and an aesthetically displeasing scalp incision can result in a psychological burden, especially for those with thin hair or baldness [7]. The aesthetic satisfaction of patients has become an important index to evaluate surgical outcomes $[8,9,12]$. With the present investigation, we observed that a regular and streamlined incision shape, consistent with the skull contour line, was more acceptable to the public's aesthetic standards.

\section{Summary}

Motivated by the need for scalp incision in decompressive hemicraniectomy, and based on our clinical experience, we applied the adjustable question mark-shaped incision design to satisfy the concerns for extensibility, blood supply, and aesthetic appearance discussed above. The scalp design is suitable for all chronic subdural procedures such as burr-hole craniotomy, mini-craniotomy, and large craniotomy. In the present case series, all the patients experienced good scalp incision healing, including the 2 patients who underwent an extension of the incision extending. Overall, 36 (97\%) patients were satisfied with the incision design itself, and only one patient was dissatisfied due to widening of the scar. Notably, most of the patients who were cosmetically self-consciousness were women, and the mean age was younger than that of the patients overall. As it happens, the majority of the patients with scalp dysfunction were also women. These results may be because women, especially younger women, are more concerned with their physical appearance than the general population. This suggests that psychological counseling before surgery is particularly important to prepare patients for the possible postoperative outcomes, especially young women, which will be our focus of the future research.

\section{Conclusion}

Taken together, the design of the scalp incision is an important issue that cannot be ignored in CSDH surgery. The adjustable question mark-shaped incision for CSDH is a fluent curved line, with a wide angle, and can be flexibly extended. It not only ensures that the procedure goes smoothly and with good wound healing, but also meets the patient's aesthetic needs. This protocol is simple, practical, and is worthy of reference for neurosurgeons, especially in primary hospitals.

\section{Abbreviations}


CSDH: chronic subdural hematoma

OR: odds ratio

Cl: confidence interval

\section{Declarations}

Founding:This research was funded by Health Commission of Jiangxi Province (20200831).

Conflicts of interest The authors declare that they have no conflict of interest.

Ethical approval This study was approved by the Ethics Committee of Second Affiliated Hospital of Nanchang University. All procedures performed in studies involving human participants were in accordance with the ethical standards of the institutional and national research committees and with the 1964 Helsinki Declaration and its later amendments or comparable ethical standards. Written informed consent was obtained from all individual participants included in the study.

Availability of data and material The datasets generated and analyzed during the current study are available from the corresponding author on reasonable request.

\section{References}

1. Huang J, Tian Y, Song Y, Hu R, Zhang S, Gong Z, Liu X, Luo H, Gao C, Wang D et al (2019) Effect of Different Factors on the Short-Term Outcome of Chinese Patients With Primary Chronic Subdural Hematoma at Different Age Groups: A Two-Center Retrospective Study. Front Aging Neurosci 11:325. https://doi.org/10.3389/fnagi.2019.00325

2. Huang J, Gao C, Dong J, Zhang J, Jiang R (2020) Drug treatment of chronic subdural hematoma. Expert opinion on pharmacotherapy 21(4):435-444. https://doi.org/10.1080/14656566.2020.1713095

3. Raghavan A, Smith G, Onyewadume L, Peck MR, Herring E, Pace J, Rogers M, Momotaz H, Hoffer SA, Hu Y et al (2020) Morbidity and Mortality After Burr Hole Craniostomy Versus Craniotomy for Chronic Subdural Hematoma Evacuation: A Single-Center Experience. World neurosurgery 134:e196-e203. https://doi.org/10.1016/j.wneu.2019.10.023

4. Yadav YR, Ratre S, Parihar V, Bajaj J, Sinha M, Kumar A: Endoscopic Management of Chronic Subdural Hematoma. Journal of neurological surgery Part A, Central European neurosurgery 2020, 81(4):330-341.https://doi.org/10.1055/s-0039-1698388

5. Jayapaul P, Lee JH, Park IS (2018) Large Scalp Defect Repair with Flap Reconstruction Using Tissue Expander After Combined Bypass in Case of Moyamoya Disease. World neurosurgery 120:185-189. https://doi.org/10.1016/j.wneu.2018.08.221 
6. Choi MG, Lee M, Suh SJ, Lee YS, Lee JH, Kang DG: Usefulness of Allogenic Acellular Dermal Matrix for Prevention of Scalp Depression after Burr Hole Trephination. Korean journal of neurotrauma 2020, 16(2):174-180.10.https://doi.org/13004/kjnt.2020.16.e34

7. Sanada Y, Yabuuchi T, Yoshioka H, Kubota H, Kato A (2015) Zigzag skin incision effectively camouflages the scar and alopecia for moyamoya disease: technical note. Neurologia medicochirurgica 55(3):210-213. https://doi.org/10.2176/nmc.tn.2014-0193

8. Stienen MN, Akeret K, Vasella F, Velz J, Jehli E, Scheffler P, Voglis S, Bichsel O, Smoll NR, Bozinov O et al (2019) COveRs to impRove AesthetiC ouTcome after Surgery for Chronic subdural haemAtoma by buRr hole trepanation (CORRECT-SCAR): protocol of a Swiss single-blinded, randomised controlled trial. BMJ open 9(12):e031375. https://doi.org/10.1136/bmjopen-2019-031375

9. Vasella F, Akeret K, Smoll NR, Germans MR, Jehli E, Bozinov O, Regli L, Stienen MN (2018) Improving the aesthetic outcome with burr hole cover placement in chronic subdural hematoma evacuation-a retrospective pilot study. Acta Neurochir 160(11):2129-2135. https://doi.org/10.1007/s00701-0183659-9

10. Pandey P, Steinberg GK (2011) Outcome of repeat revascularization surgery for moyamoya disease after an unsuccessful indirect revascularization. Clinical article. Journal of neurosurgery 115(2):328-336. https://doi.org/10.3171/2011.3.JNS101908

11. Soto JM, Feng D, Sun H, Zhang Y, Lyon KA, Liang B, Reed LK, Huang JH: Novel Decompressive Hemicraniectomy Technique for Traumatic Brain Injury: Technical Note. World neurosurgery 2021, 146:15-19.https://doi.org/10.1016/j.wneu.2020.10.054

12. Funk W, Podmelle F, Guiol C, Metelmann HR: Aesthetic satisfaction scoring - introducing an aesthetic numeric analogue scale (ANA-scale). Journal of cranio-maxillo-facial surgery: official publication of the European Association for Cranio-Maxillo-Facial Surgery 2012, 40(5):439442.https://doi.org/10.1016/j.jcms.2011.07.018

\section{Figures}




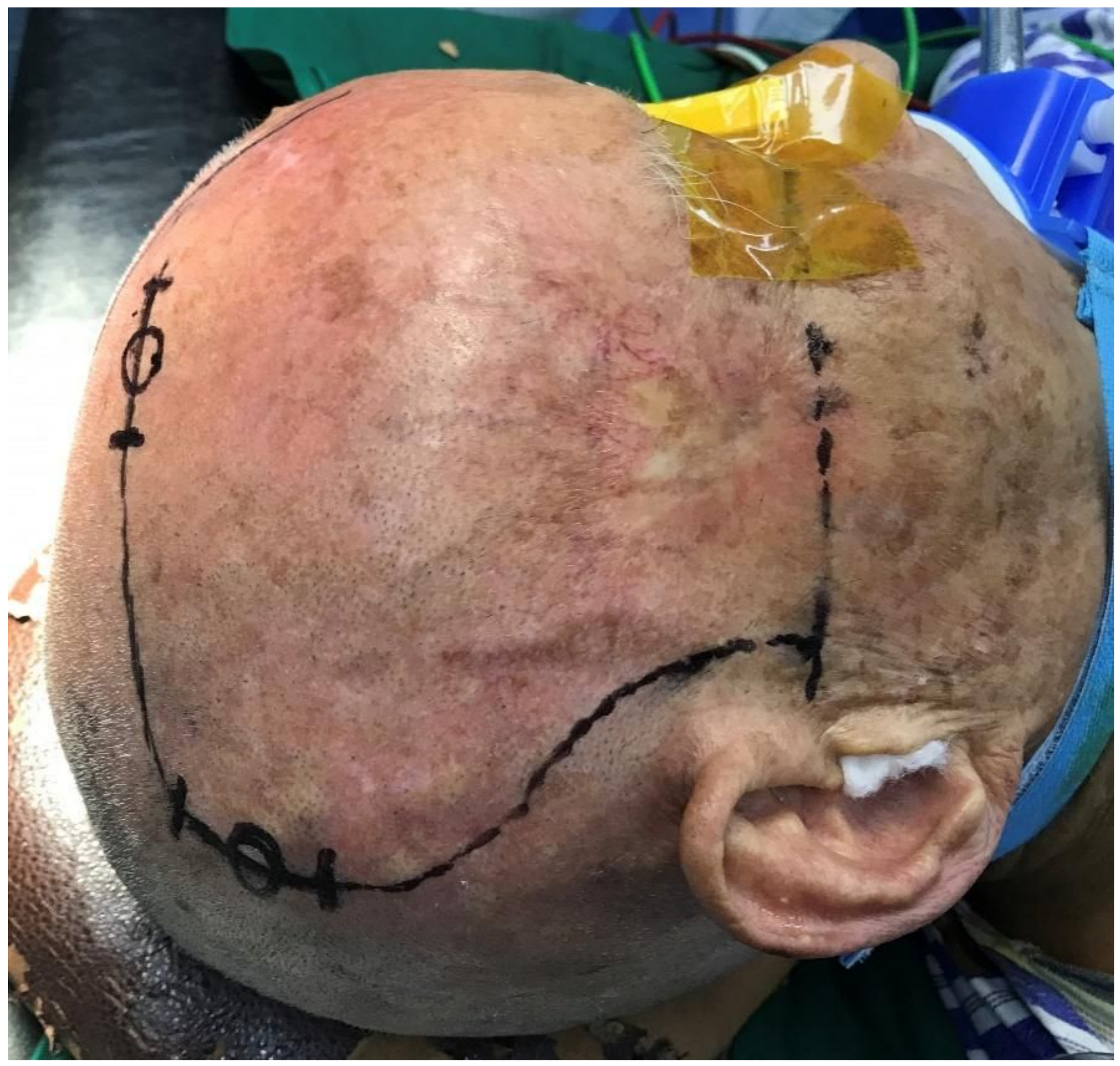

\section{Figure 1}

A similar question-mark line which is used in decompressive hemicraniectomy usually is drawn on the frontal to parietal to temporal scalp. The anterior half of the question-mark line is roughly parallel to the superior temporal line, and its front end intersects the scalp projection of the coronal suture and ends within the hairline of the forehead. The posterior end of the question-mark line (the arc) is located at the parietal tubercle or about 0.5 - to 1-centimeter anteroinferior to the parietal tubercle. 


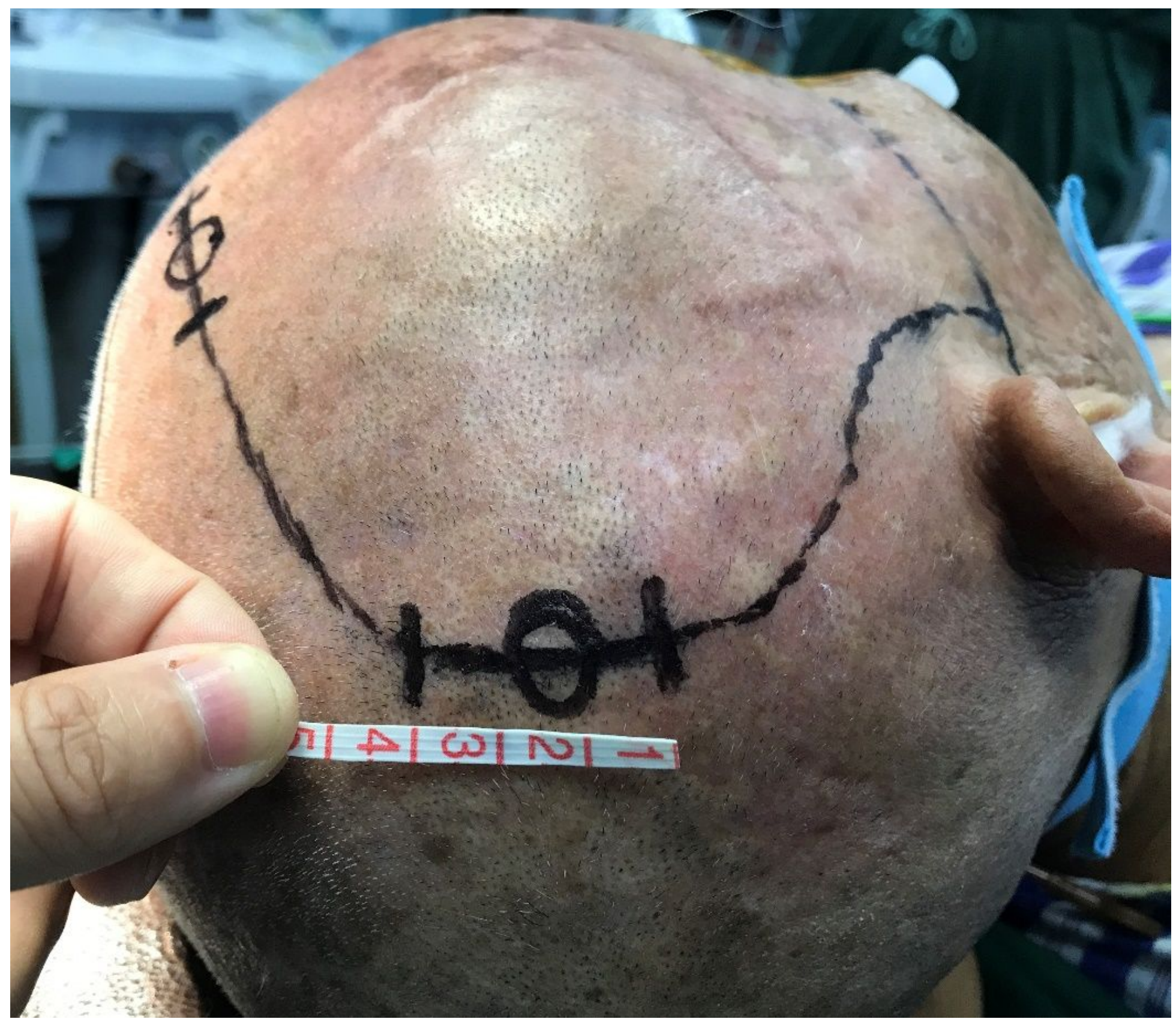

Figure 2

For single burr-hole craniotomy, a 2- to 3-centimeter incision was made along the arc portion of the line (i.e., the posterior end of the question-mark). 


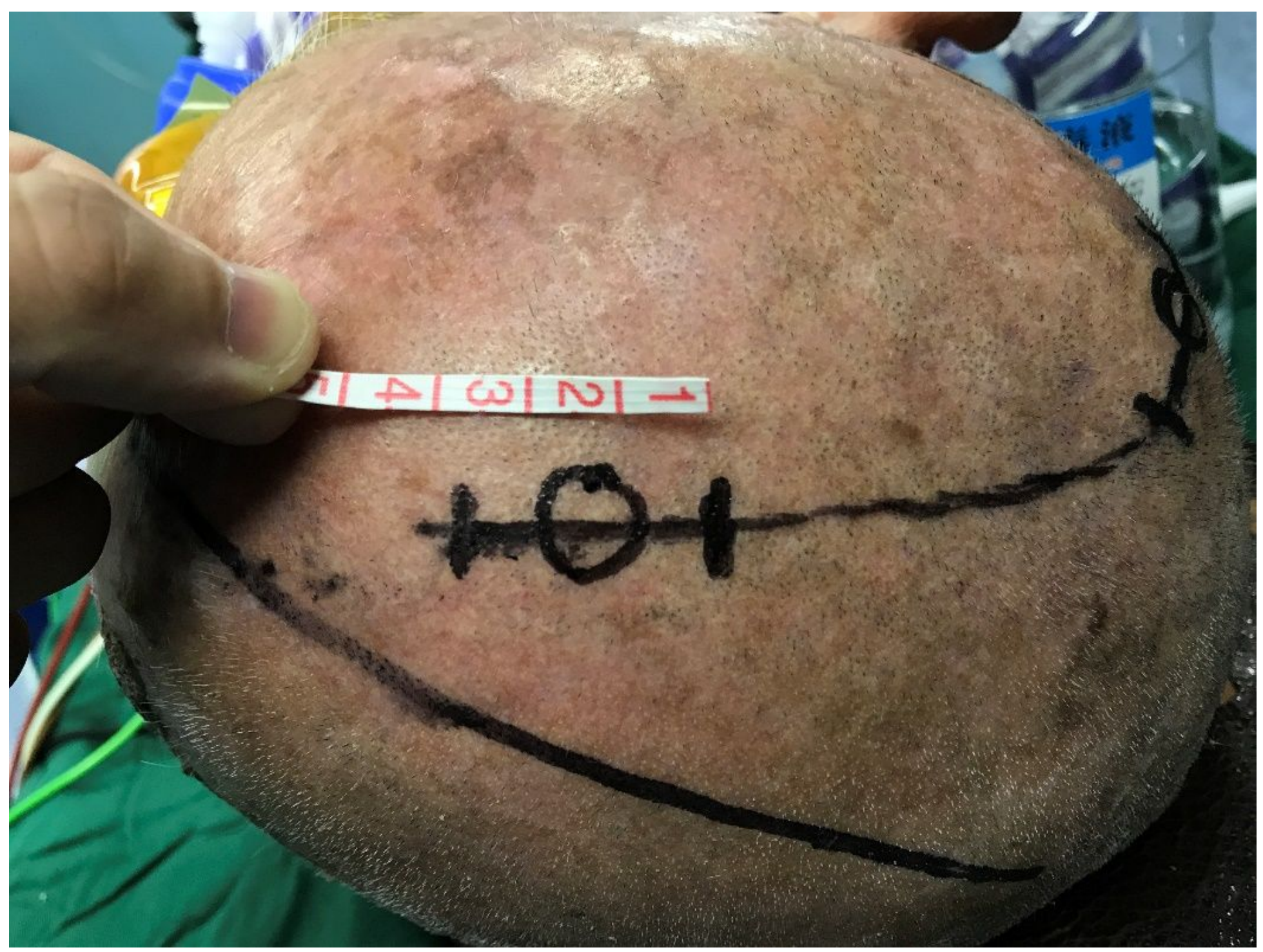

\section{Figure 3}

In double burr-hole craniotomy, the second incision is placed at the intersection of the question-mark line and the scalp projection of the coronal suture, or slightly anterior to the coronal suture. 


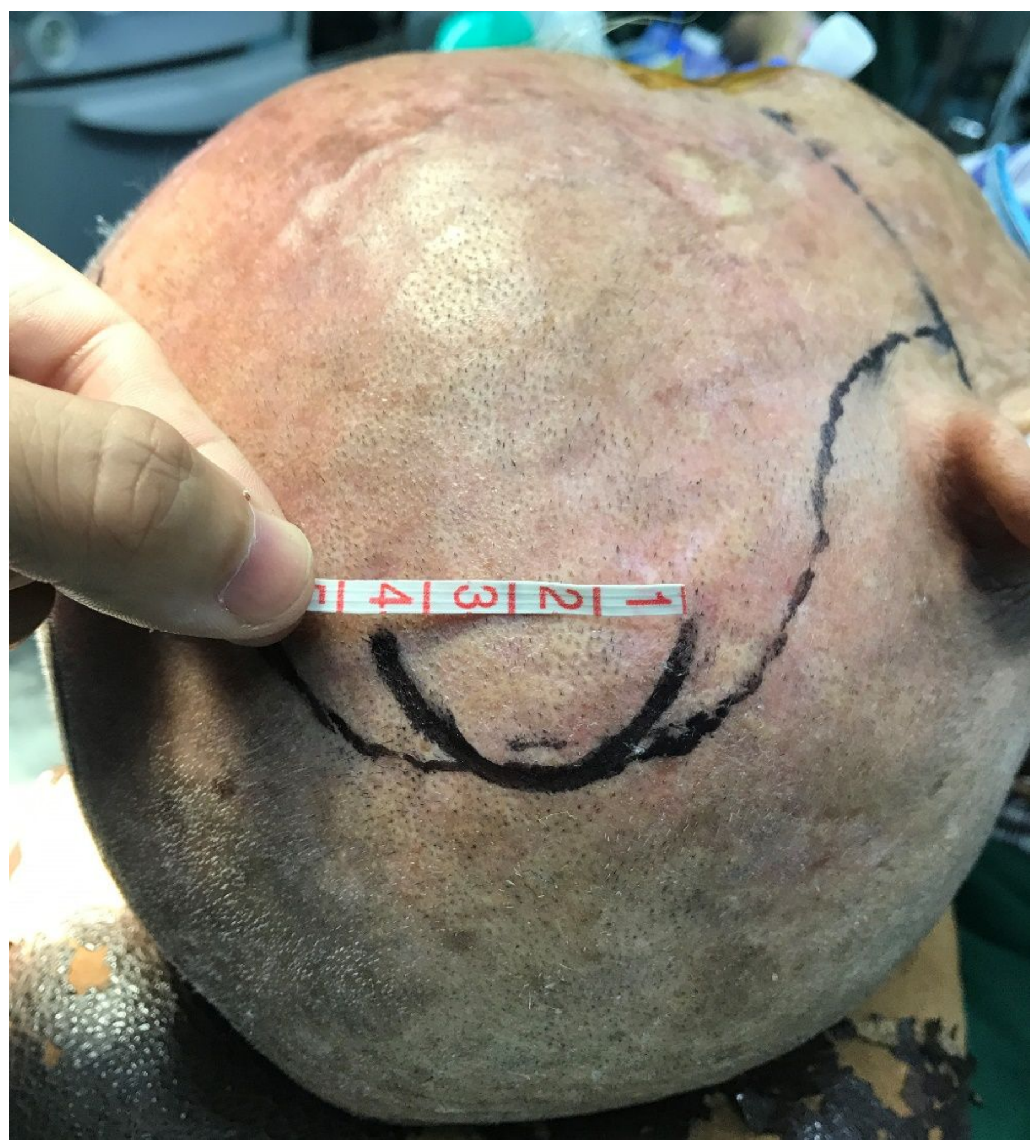

Figure 4

Mini-craniotomy. The incision is located on the arc of the question-mark line, and the radian of the arc can be reduced moderately to make the exposed skull about 2.5- to 3-centimeters in diameter. 


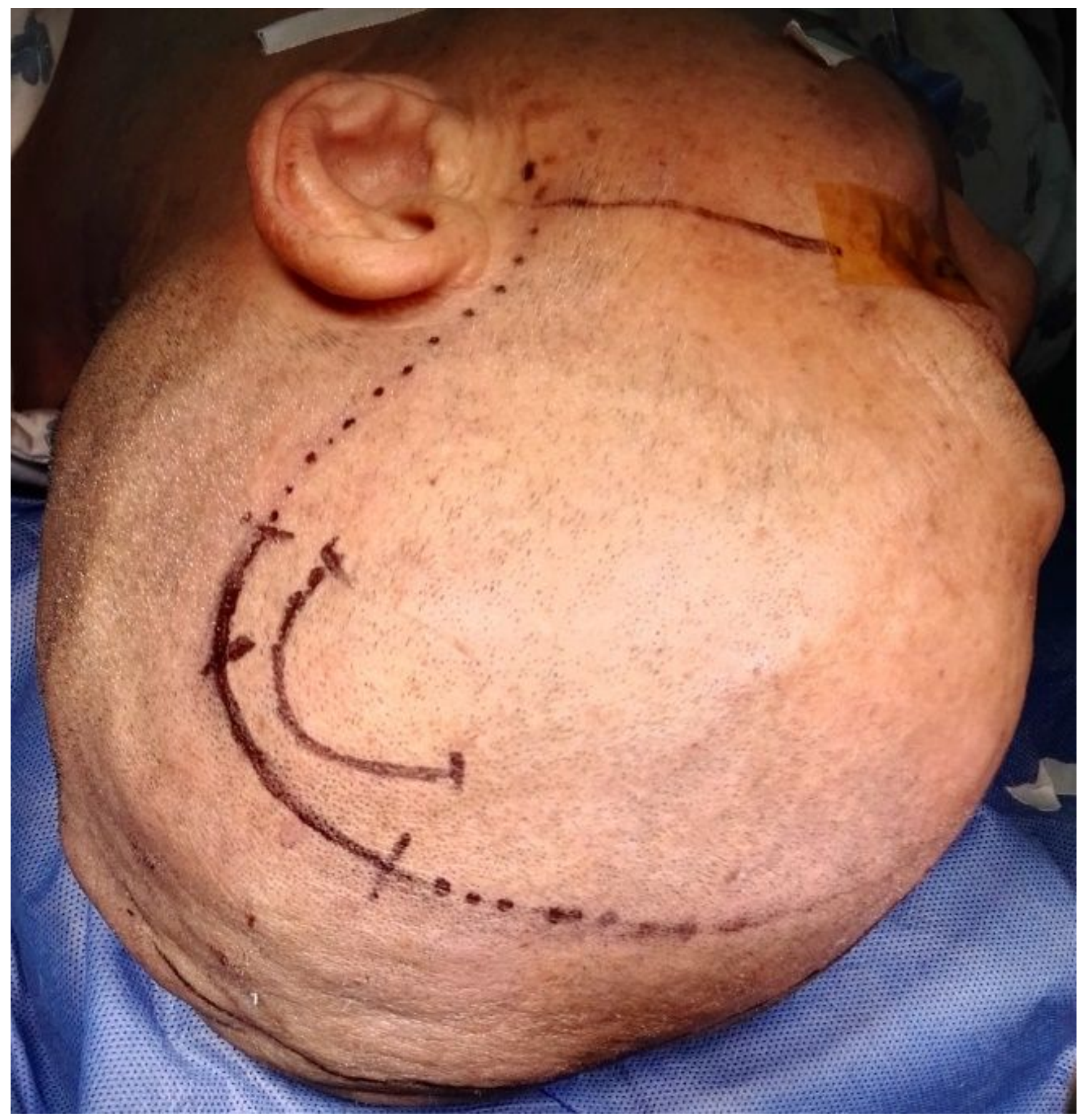

\section{Figure 5}

Sometimes the arc portion of the line can be appropriately moved forward for the incision. 